\title{
The Evolution of Hemophilia Care: Clinical and Laboratory Advances, Opportunities, and Challenges
}

\author{
Alice Trinchero $^{1,2}$ Michelle Sholzberg ${ }^{3}$ Davide Matino ${ }^{4,5}$ \\ ${ }^{1}$ Center for Thrombosis and Hemostasis, University Medical Center \\ Mainz, Johannes Gutenberg University, Mainz, Germany \\ 2 Department of Clinical Oncology and Hematology, University \\ Hospital Zurich, Zurich, Switzerland \\ ${ }^{3}$ Hematology/Oncology, St. Michael's Hospital, Li Ka Shing \\ Knowledge Institute, University of Toronto, Toronto, Ontario, \\ Canada \\ ${ }^{4}$ Department of Medicine, Hamilton, Ontario, Canada \\ ${ }^{5}$ Department of Medicine, McMaster University, Hamilton, Ontario, \\ Canada
}

\author{
Address for correspondence Davide Matino, MD, MSc, Division of \\ Hematology and Thromboembolism, Department of Medicine, \\ McMaster Children's Hospital, 1280 Main Street West, Hamilton, \\ Ontario, Canada (e-mail: matinod@mcmaster.ca).
}

Hämostaseologie 2020;40:311-321.

\begin{abstract}
Keywords

- hemophilia A/B

- gene therapy

- factor VIII

- factor IX

Hemophilia $A(H A)$ and $B(H B)$ are X-linked bleeding disorders caused by mutations in the $\mathrm{F} 8$ or $\mathrm{F} 9$ gene that result in the absence, or reduced activity, of the corresponding clotting factor. The severity of bleeding and related complications is proportional to the amount of residual circulating functional factor. The development of a safe and effective hemophilia treatment lasted several decades and has been mainly based on clotting factor replacement. Advances in the engineering and manufacturing of clotting concentrates have led to the widespread availability of extended half-life products that reduced the number of intravenous infusions needed to achieve adequate trough levels. The recent development of new nonfactor replacement treatments and biotechnology techniques has offered therapeutic alternatives for hemophilia patients with and without inhibitors. These are characterized by an easier route of administration, low immunogenicity, and, regarding gene therapy and cellbased treatments, potential long-term protection from bleeding after a single treatment course. In this review, we analyze recent progresses in the management of hemophilia and discuss opportunities and challenges.
\end{abstract}

\section{Introduction}

Hemophilia A (HA) and B (HB) are X-linked bleeding disorders caused by mutations in the F8 or F9 gene that result in the absence, or reduced activity, of the corresponding clotting factor. ${ }^{1}$ The severity of clinical manifestations is proportional to the amount of residual circulating functional factor. In severe cases (clotting factor level $<0.01 \mathrm{IU} / \mathrm{mL}$ ), spontaneous bleeds are an integral part of the clinical picture. These are typically hemarthrosis (intra-articular bleeding). Muscle bleeding, soft tissue hemorrhage, and intracranial hemor- rhages (ICHs) are also frequently described. ${ }^{2}$ Repeated hemarthroses lead to a hypertrophic synovitis characterized by an increased production of soluble mediators of inflammation with progressive cartilage degradation and bone damage, chronic pain, and irreversible joint function impairment. $^{3}$ On the other side of the severity spectrum, patients with higher levels of circulating factors and moderate or mild manifestations of disease may experience bleeding only after traumatic events or surgical procedures. ${ }^{4}$ Moderate hemophilia is defined for FVIII:C levels of 0.01 to $<0.05 \mathrm{IU} / \mathrm{mL}$. Mild HA is characterized by either FVIII:C levels received

February 12, 2020

accepted after revision

May 11, 2020 (c) 2020 Georg Thieme Verlag KG Stuttgart · New York
DOI https://doi.org/

10.1055/a-1175-6530. ISSN 0720-9355. 
of $<0.4 \mathrm{IU} / \mathrm{mL}$ or FVIII:C $>0.4 \mathrm{IU} / \mathrm{mL}$ plus evidence of a listed F8 DNA-mutation associated with $\mathrm{HA}$ and FVIII:C $<0.4 \mathrm{IU} / \mathrm{mL}$ or a family member with the same DNA change and FVIII:C $<0.4 \mathrm{IU} / \mathrm{mL}^{4}$

The development of a safe and effective hemophilia treatment required several decades of efforts and has been mainly based on replacement of the missing clotting factor. Barriers to adequate therapy remain and limit the achievement of an adequate bleeding control and prevention of joint deterioration, such as difficult venous access-particularly in infantsthe high frequency of intravenous treatment, and the development of an immune response toward the therapeutically administered clotting factor. The latter, neutralizing antibodies to exogenous factor denominated "inhibitors," is a major issue in hemophilia treatment that precludes the possibility of an appropriate prophylactic treatment to these patients.

Advances in the engineering and manufacturing of clotting concentrates haveled to the widespread availability of extended half-life (EHL) products that reduced the number of intravenous infusions needed to achieve adequate trough levels. The recent development of new non factor replacement treatments and biotechnology advances hasoffered therapeutic alternatives for hemophilia patients with and without inhibitors, characterized by an easier route of administration, low immunogenicity, and, regarding gene therapy and cell-based treatments, potential long-term protection from bleeding after a single treatment course.

In this review, we analyze recent progresses in the management of hemophilia and discuss opportunities and challenges.

\section{The Evolution of Factor Replacement Therapy and Its Impact on Life Expectancy}

The treatment of hemophilia has been based on coagulation factor replacement for the past 60 years. In the 1960s, novel plasma fractionation techniques were instrumental for the extraction of high-concentration factor VIII (FVIII) and von Willebrand factor from the precipitate of frozen plasma. Cryoprecipitates became the first effective therapy of hemophilia-related bleeding. ${ }^{5}$ The industrial manufacturing of plasma-derived highly enriched FVIII and factor IX (FIX) concentrates broadened the availability of supplies. This translated into an earlier control of hemorrhages and the opportunity to opt for home treatment, ${ }^{6}$ but also favored the transition from an "on demand" treatment to routine bleeding prophylaxis. ${ }^{1}$ The first national programs of specialized hemophilia treatment centers were launched with the objective to provide a specialized, comprehensive care.

In these first years, life expectancy of hemophilia patients dramatically increased ( - Table 1). In the late 1950s, half of the patients with hemophilia ( $\mathrm{PwH}$ ) would have died by the age of 19 years, whereas the median life expectancy reached approximately 50 years in Northern Europe and America in the early 1980 s. $^{7,8}$ This was accompanied by an improvement in quality of life (QoL), particularly among severe patients, ${ }^{9-11}$ and a reduction of the burden of hemophilia arthropathy. ${ }^{12}$ These positive trends were abruptly interrupted in the 1980s: the human immunodeficiency virus (HIV) and non-A hepatitis viruses spread among the hemophilic population, as clotting factors concentrates were produced by pooling the plasma from thousands blood donors. ${ }^{13}$ Routine viral testing of the blood donors would have been introduced only in the year 1985. Novel purification methods and viral inactivation/removal techniques for the production of plasma-derived concentrates were progressively implemented to minimize the risk of new infections. ${ }^{14,15}$ In the United States, the median age of death fell to 35 years in the years 1993 to 1995 at the peak of the HIV epidemic with acquired immune deficiency syndrome (AIDS) recorded as the immediate or underlying cause in more than $60 \%$ of deaths in PwH (-Table 1). ${ }^{16,17}$

F8 was cloned in $1984,{ }^{18}$ paving the way to the production of recombinant FVIII-concentrates, which consisted of three generations of products: (1) animal-derived proteins with human serum albumin added, (2) human-derived proteins without albumin, and (3) manufactured FVIII only. ${ }^{19}$ The last two generations included concentrates lacking the FVIII Bdomain, which appeared to be unnecessary for FVIII-coagulant activity, but rather involved in intracellular trafficking, secretion, and possibly implications for immunogenicity. ${ }^{20}$

The availability of safe and effective replacement therapy with factor concentrates substantially improved the care of hemophilia patients. However, the formation of inhibitory alloantibodies against the infused FVIII and FIX is a major complication of the treatment with clotting factor concentrates. Inhibitors develop in 20 to $40 \%$ and 3 to $10 \%$ of patients affected by severe $\mathrm{HA}$ and $\mathrm{HB}$, respectively, mostly within the first 50 , but also up to 100 to 500 exposure days. ${ }^{21,22} \mathrm{~A}$ lower amount of patients affected by mild or moderate HA, namely 3 to $13 \%$, could also develop an immune response to the infused clotting factor.

The presence of inhibitors causes ineffectiveness of the treatment with a consequent increase in morbidity and mortality. ${ }^{23,24}$ Agents bypassing the inhibitors activity, such as activated prothrombin complex concentrate (aPCC) and recombinant activated factor VII ( $r F V I I a)$, have been available since the 1970s to 1980s, albeit their efficacy is lower than that of FVIII concentrates, ${ }^{25}$ and are characterized by higher costs and treatment burden. ${ }^{26}$

The modern approach to factor replacement therapy consisted of (1) "on demand" treatment to treat acute bleeding; (2) short-term prophylaxis before surgical or invasive procedures; (3) primary long-term prophylaxis starting before the age of 2years and after no more than one joint bleed, given at a fixed dose mostly two to three times per week; (4) routine or intermittent treatment in the context of a secondary or tertiary prophylaxis to prevent recurrent bleeding events and their complications. ${ }^{27}$ Evidence from clinical trials and a recent systematic review illustrated the reduction of bleeding rate if a secondary ${ }^{28-31}$ or primary prophylaxis ${ }^{32}$ was in place, as compared with an on-demand treatment. ${ }^{33}$ The benefits are larger if the prophylaxis is started prior to development of target joints damage ${ }^{34}$ and are particularly pronounced in terms of ICH reduction. ${ }^{35}$

The increased trend in life expectancy and the improved QoL observed over the past 20 years among PwH is due, at least in part, to the high-quality factor concentrates and progress in the management of blood-borne viral infections, 
Table 1 Time trends in life expectancy and underlying causes of death in persons with hemophilia A and B

\begin{tabular}{|c|c|c|c|c|}
\hline Country & Years & $\begin{array}{l}\text { Persons with } \\
\text { hemophilia }\end{array}$ & $\begin{array}{l}\text { Median life expectancy or } \\
\text { median age at death (y) }\end{array}$ & Main causes of death \\
\hline Sweden 7,8 & $1831-1980$ & Severe hemophilia & $\begin{array}{l}\text { Median life expectancy } \\
\text { 1831-1920: } 11 \text { y } \\
\text { 1921-1940: } 23 \text { y } \\
\text { 1941-1960: } 36 \text { y } \\
\text { 1961-1980: } 57\end{array}$ & Hemorrhage (ICH 33\%) \\
\hline Netherlands ${ }^{37}$ & 1973-1992 & Severe hemophilia & $\begin{array}{l}\text { Median life expectancy } \\
\text { 1973-1986: } 63 \text { y } \\
\text { 1986-1992: } 61 \text { y }\end{array}$ & $\begin{array}{l}\text { 1973-1986: cancer 35\% } \\
\text { 1986-1992: AIDS } 27 \%\end{array}$ \\
\hline United States ${ }^{16}$ & 1993-1995 & $\mathrm{PwH}, \mathrm{HIV}-$ & $\begin{array}{l}\text { Median life expectancy at } \\
\text { birth } 39 \mathrm{y}(\mathrm{PwH}), 64 \mathrm{y}(\mathrm{HIV}-) \\
\text { Median age at death } \\
35 \mathrm{y}(\mathrm{PwH}), 67 \mathrm{y}(\mathrm{HIV}-)\end{array}$ & $\begin{array}{l}\text { AIDS } 64 \% \text { ( } 53 \% \text { final cause, } \\
12 \% \text { underlying cause) }\end{array}$ \\
\hline United States ${ }^{17}$ & 1979-1998 & Hemophilia A & $\begin{array}{l}\text { Median age at death } \\
\text { 1979-1982: } 50.6 \mathrm{y} \\
\text { 1987-1990: } 44 \mathrm{y} \\
\text { 1995-1998: } 50.8 \mathrm{y}\end{array}$ & $\begin{array}{l}\text { 1979-1982: circulatory } 43 \% \\
\text { 1987-1998: HIV 57-47\% }\end{array}$ \\
\hline Netherlands ${ }^{38}$ & $1972-2001$ & Severe hemophilia & $\begin{array}{l}\text { Median life expectancy } \\
\text { 1972-1985: } 63 \text { y } \\
\text { 1985-1992: } 61 \text { y } \\
\text { 1992-2001: } 59 \text { y }\end{array}$ & $\begin{array}{l}\text { 1973-1986: hemorrhage } 47 \% \\
\text { 1986-1992: AIDS 27\% } \\
\text { 1992-2001: AIDS 26\%, } \\
\text { hepatitis C 22\% }\end{array}$ \\
\hline United Kingdom ${ }^{39}$ & 1977-1999 & $\begin{array}{l}\text { Severe, moderate, } \\
\text { or mild hemophilia }\end{array}$ & $\begin{array}{l}\text { Median life expectancy } \\
\text { 1999: } 63 \text { y (severe), } 75 \text { y } \\
\text { (moderate or mild) }\end{array}$ & $\begin{array}{l}\text { Hemorrhage (particularly ICH), } \\
\text { and liver disease }\end{array}$ \\
\hline Italy ${ }^{36}$ & 1990-2007 & $\begin{array}{l}\text { Severe hemophilia A, } \\
\text { severe hemophilia B }\end{array}$ & $\begin{array}{l}\text { Median age at death } \\
39 \text { y }(\mathrm{HA}), 35 \text { y }(\mathrm{HB}) \\
\text { Median life expectancy } \\
\text { 1995: } 59.2 \text { y }(\mathrm{HA}), 40.3 \text { y }(\mathrm{HB}) \\
\text { 2005: } 64.5 \text { y }(\mathrm{HA}), 66.3 \text { y }(\mathrm{HB})\end{array}$ & $\begin{array}{l}\text { 1980-1999: AIDS 56-60\% } \\
\text { 2000-2007: hemorrhage } 27 \%\end{array}$ \\
\hline United States ${ }^{42}$ & 2007 & $\mathrm{PwH}$ & $\begin{array}{l}\text { Median age at death } \\
68 \mathrm{y}\end{array}$ & Sepsis 38\% \\
\hline Sweden $^{43}$ & 1968-2009 & $\mathrm{PwH}$ & $\begin{array}{l}\text { Median age at death } \\
\text { 1981-1990: } 46 \mathrm{y} \\
\text { 1991-2000: } 41 \mathrm{y} \\
\text { 2001-2008: } 56 \mathrm{y}\end{array}$ & $\begin{array}{l}\text { Cancer } 22 \text { and } 24 \% \text { (total and HIV-) } \\
\text { AIDS } 8 \text { and 31\% (total and severe) }\end{array}$ \\
\hline Korea $^{45}$ & $1991-2012$ & $\mathrm{PwH}$ & $\begin{array}{l}\text { Median life expectancy } \\
\text { 2010: } 69 \text { y }\end{array}$ & Hemorrhage 52.6\% (ICH 36.5\%) \\
\hline Taiwan $^{40}$ & 1997-2009 & $\mathrm{PwH}$ & $\begin{array}{l}\text { Median age at death } \\
\text { 1997-2004: } 36.3 \mathrm{y} \\
\text { 2005-2009: } 51.8 \mathrm{y}\end{array}$ & Not available \\
\hline United States $^{44}$ & $1998-2011$ & $\begin{array}{l}\text { PwH with or } \\
\text { without inhibitors }\end{array}$ & $\begin{array}{l}\text { Median age at death } \\
39.5 \text { y (with inhibitors) } \\
40.4 \text { y (without inhibitors) }\end{array}$ & $\begin{array}{l}\text { Hemophilia-related } \\
42 \% \text { (inhibitors) } \\
\text { Liver disease-related } \\
32 \% \text { (no inhibitors) }\end{array}$ \\
\hline Netherlands ${ }^{41}$ & $1980-2011$ & $\begin{array}{l}\text { Nonsevere } \\
\text { hemophilia A }\end{array}$ & $\begin{array}{l}\text { Median age at death } \\
\text { 1980-1989: } 32 \text { y } \\
\text { 1990-1999: } 59 \text { y } \\
\text { 2000-2010: } 69 \text { y }\end{array}$ & $\begin{array}{l}\text { 1980-1989: ICH } 20 \% \\
\text { 1990-1999: AIDS } 17 \% \\
\text { 2000-2010: cancer 32\% }\end{array}$ \\
\hline $\operatorname{Iran}^{46}$ & $1975-2015$ & $\begin{array}{l}\text { Hemophilia A, } \\
\text { hemophilia B }\end{array}$ & $\begin{array}{l}\text { Median age at death } \\
17.2 \mathrm{y}, 33.1 \mathrm{y}\end{array}$ & Hemorrhage 65-25\% \\
\hline
\end{tabular}

Abbreviations: AIDS, acquired immune deficiency syndrome; HA, hemophilia A; HB, hemophilia B; HIV, human immunodeficiency virus; ICH, intracerebral hemorrhage; PwH, person with hemophilia.

notably HIV and hepatitis ( - Table 1). ${ }^{7,14,16,17,36-46}$ However, the mortality due to ICH was not remarkably affected, especially in patients without inhibitors and in moderate/ mild hemophilia patients. ${ }^{39,47,48}$

Recombinant FVIII and FIX concentrates with EHL have been developed based on novel techniques, such as (glycol)
PEGylation, Fc fusion, or sequence modification. These products are characterized by higher plasma levels and a reduced frequency of administration. ${ }^{49}$ Due to a wider access to highquality prophylaxis and a comprehensive care of associated complications, PwH are now able to achieve previously unattainable life expectancy, close to that of general population in 
many countries. Treatment goals have evolved from simply preventing early death to decreasing spontaneous and subclinical bleeding, prevent disability and late-onset diseases (e.g., cardiovascular diseases, cancer), and improve QoL.

\section{Gaps of Knowledge}

Although pharmacokinetic algorithms to individualize primary and secondary prophylaxis are in place ${ }^{50}$ and novel products characterized by a prolonged half-life became available, hemophilic arthropathy is not adequately prevented over lifetime; similarly, the risk of ICH among PwH exceeds that of the general nonhemophilic population. ${ }^{51-53}$ A conservative prophylactic approach based on the historical threshold of FVIII $\geq 1 \mathrm{IU} / \mathrm{dL}$ derived from prior studies on cryoprecipitates $^{54,55}$ has been recently questioned. A higher threshold of FVIII (e.g., 15\% or higher) has been proposed and may be reasonable for selected patients. ${ }^{56}$ Not only the dosing, but also the timing of treatment initiation may be critical. An earlier prophylaxis, e.g., starting at the time of diagnosis (at birth for severe hemophilia), is crucial for maintaining healthy joints and preventing $\mathrm{ICH}$ during the first months of life. ${ }^{33}$

A stratification strategy has the goal to predict the occurrence of inhibitory alloantibodies and the outcome of immunotolerance induction (ITI), the treatment of choice to eradicate the inhibitors. Several genetic (ethnicity, F8-mutations, major histocompatibility complex) and acquired (number of FVIII-exposure days, age at first exposure to FVIII concentrate, type of concentrate) factors influence the development and course of inhibitors, ${ }^{21,57,58}$ including potential differences in immunogenicity across factor concentrates in previously untreated patients. ${ }^{59,60}$

The rising of late-onset diseases (cardiovascular, metabolic and renal disorders, cancer) following the improved life expectancy in the hemophilia population represents nowa- days a new challenge for patients and physicians, whereas the reduction in hepatitis $\mathrm{C}$ virus (HCV) and HIV infections and available curative therapies for HCV infection lead to a new generation of HIV- and HCV-free patients. This will likely affect future trends in morbidity and mortality.

\section{Novel Therapies for Hemophilia}

New treatments have been designed that are less burdensome for the patients and more effective, irrespective of the presence of inhibitors. These nonfactor therapeutic products are long-acting and can be administered subcutaneously. Their mechanisms aim at rebalancing the hemostatic defect by targeting some of the natural anticoagulant pathways that regulate hemostasis (e.g., fitusiran, concizumab), by enhancing coagulation (emicizumab), or providing a long-term solution to factor deficiency (gene therapy).

\section{Emicizumab}

Emicizumab is a humanized bispecific monoclonal antibody binding FIXa and FX and thus, as a cofactor mimetic, replacing the function of the missing FVIIIa and allowing for the formation of a functional tenase complex. ${ }^{61}$ The relatively weak binding affinities reduce the interference within the coagulation cascade and permits the release of FXa from the complex and participate in the downstream reactions. ${ }^{49}$ Emicizumab does not have any homology with FVIII and therefore is not inhibited by the presence of neutralizing anti-FVIII antibodies. Additionally, being a monoclonal antibody has the advantage of subcutaneous administration and long half-life. A phase 3 , multicenter trial was conducted to assess once-weekly subcutaneous emicizumab prophylaxis in patients with HA and FVIII inhibitors (HAVEN 1; - Table 2). ${ }^{62}$ Subjects randomized to emicizumab (treated weekly with $3 \mathrm{mg} / \mathrm{kg}$ as loading doses for 4 weeks and then followed by weekly injections of $1.5 \mathrm{mg} / \mathrm{kg}$ )

Table 2 Novel treatment options for the treatment of hemophilia: features and trials data

\begin{tabular}{|c|c|c|c|c|}
\hline Drug & Study & Regimen & ABR (median) & Zero bleeding rates \\
\hline \multirow[t]{9}{*}{ Emicizumab } & \multirow[t]{2}{*}{ HAVEN $1^{62}$} & 1.5 mg/kg per wk & 2.9 & $63 \%$ \\
\hline & & No prophylaxis & 23.3 & $6 \%$ \\
\hline & \multirow[t]{3}{*}{ HAVEN $2^{63}$} & $1.5 \mathrm{mg} / \mathrm{kg}$ per wk & 0.3 & $77 \%$ \\
\hline & & $3.0 \mathrm{mg} / \mathrm{kg}$ every $2 \mathrm{wk}$ & 0.2 & $90 \%$ \\
\hline & & $6 \mathrm{mg} / \mathrm{kg}$ every $4 \mathrm{wk}$ & 2.2 & $60 \%$ \\
\hline & \multirow[t]{3}{*}{ HAVEN $3^{64}$} & $1.5 \mathrm{mg} / \mathrm{kg}$ per wk & 1.5 & $50 \%$ \\
\hline & & $3.0 \mathrm{mg} / \mathrm{kg}$ every $2 \mathrm{wk}$ & 1.3 & $40 \%$ \\
\hline & & No prophylaxis & 38.2 & $0 \%$ \\
\hline & HAVEN 4 & $6 \mathrm{mg} / \mathrm{kg}$ every $4 \mathrm{wk}$ & 4.5 & \\
\hline Fitusiran & $\begin{array}{l}\text { OLE } \\
\text { (presented at } \\
\text { ISTH 2017) }\end{array}$ & $50 / 80 \mathrm{mg}$ once a month & 0 & $22 / 33$ \\
\hline \multirow[t]{3}{*}{ Concizumab } & \multirow[t]{2}{*}{ explorer $4^{72}$} & \multirow{3}{*}{$\begin{array}{l}0.15 \mathrm{mg} / \mathrm{kg} \text { with sequential } \\
\text { dose escalation }(0.20,0.25) \\
\text { in case of } 3 \text { spontaneous } \\
\text { bleedings or more }\end{array}$} & HAwl: 3 & \\
\hline & & & HBwl: 5.9 & \\
\hline & explorer5 $^{72}$ & & HA: 4.5 & \\
\hline
\end{tabular}

Abbreviations: ABR, annualized bleeding rate; HAwl, hemophilia A patients with inhibitor; HBwl, hemophilia B patients with inhibitor; ISTH, International Society on Thrombosis and Haemostasis. 
were compared with a control group treated with on-demand bypassing agents, showing a difference in the annualized bleeding rate (ABR, $87 \%$ ) in favor of emicizumab prophylaxis. In the HAVEN 2 trial, pediatric patients with severe HA with inhibitors were treated with emicizumab. Prophylaxis with once-weekly subcutaneous emicizumab resulted in a very low bleeding rate in children with HA and FVIII inhibitors. The authors reported a lower bleeding rate with once-weekly emicizumab compared with prior standard prophylaxis, and the majority of participants (77\%) had no treated bleeding events. ${ }^{63}$

The efficacy of prophylaxis with emicizumab in HA without inhibitors has been evaluated in the HAVEN 3 trial using weekly or biweekly dosing. The results demonstrated a $68 \%$ reduction in treated bleeds compared with prior FVIII prophylaxis. ${ }^{64}$ This finding supports the concept that steadystate hemostatic correction with nonfactor therapy can provide a protection possibly superior to the standard prophylaxis, which is associated with peaks and troughs of factor activity that can be more or less pronounced depending on frequency and dose of the treatment.

\section{Fitusiran}

Fitusiran is a recently developed small-interfering RNA (siRNA) that acts by targeting and binding antithrombin (AT) messenger RNA, altering AT gene translation and blocking protein synthesis. AT can inactivate FXa and thrombin, and silencing AT causes a significant hypercoagulable state. ${ }^{65}$ Because of the central role of thrombin and FXa in the physiological development and stability of clots, strategies that target AT are particularly attractive. The reduction of circulating AT can improve thrombin generation and has the potential to attenuate the bleeding symptoms. ${ }^{66}$ In fact, in hemophilic mice, the targeting of AT via siRNA resulted in protection from bleeding and prolonged the lifespan of the animals. The same biotechnological approach, acting on coagulation gene transcription via siRNA, has been shown to be effective in vivo in several contexts. ${ }^{67}$ A phase 1 dose-escalation study was conducted in healthy volunteers and 25 hemophilic patients without inhibitors, showing that the subcutaneous administration of fitusiran could be a promising approach. ${ }^{68}$ These studies also showed the potential for monthly dosing, and the applicability to patients with HA and HB with and without inhibitors together with a low volume was used for the subcutaneous administration. Doses of 50 and $80 \mathrm{mg}$ once a month in 14 inhibitor patients and 19 noninhibitor patients were tested. ${ }^{69}$ The interim data analysis at a median of 13 months of treatment that was presented at the 2017 International Society on Thrombosis and Haemostasis congress demonstrated a significant decrease in AT levels. Of note, in treated patients with inhibitors, the median $A B R$ was 0 ( 38 before the study), with 22/33 of patients reporting no spontaneous bleeds. Breakthrough bleeds could be successfully controlled with factor replacement therapy or bypassing agents. Phase 3 trials enrolling severe HA or HB patients, with or without inhibitors, pediatrics, and adults to further assess the safety and efficacy of fitusiran are ongoing. A fatal severe adverse event, a cerebral vein thrombosis, occurred in a patient initially misdiagnosed as a cerebral bleeding and treated with a FVIII concentrate. After a temporary trial suspension and the development of risk-mitigation strategies to guide therapy with FVIII or bypassing agents when treating breakthrough bleeds, these trials have been reopened. ${ }^{70}$

\section{Anti-TFPI Antibodies}

Tissue factor pathway inhibitor (TFPI) downregulates the initiation of coagulation and consists of three Kunitz-type protease inhibitor domains. It mediates the inhibition of factor VIIa/tissue factor/factor Xa (FVIIa/TF/FXa), thus inhibiting the extrinsic pathway. The K1 domain can inhibit FVIIa, the K2 domain can inhibit FXa, and the K3 domain binds protein $\mathrm{S}$ (PS). The rationale of targeting TFPI is that blocking TFPI inhibition may restore TF/FVIIa-mediated FXa generation to enhance in vivo hemostasis significantly. Experiments conducted in hemophilia animal models have demonstrated that the inhibition of TFPI can correct the bleeding tendency and reduce blood loss with an effect comparable to rFVIIa. ${ }^{71}$

Several clinical trials with monoclonal antibodies targeting TFPI have been started in hemophilia patients with and without inhibitors. As for emicizumab and fitusiran, also new therapies, anti-TFPI antibodies havethe potential to be given subcutaneously and at weekly intervals. Three phase 2 trials in HA and HB patients with (HAwI andHBwI) and without inhibitors (HA) have been completed. In the Explorer 4 and Explorer 5 trials, ${ }^{72}$ the estimated ABRs in HAwI and HBwI were lower versusHA: 3.0 and 5.9 versus 7.0, respectively. Most inhibitor patients (88.2\%) did not escalate the starting dose of $0.15 \mathrm{mg} / \mathrm{kg}$, with potential dose escalation to 0.20 and $0.25 \mathrm{mg} / \mathrm{kg}$ in the case of three spontaneous bleeding episodes or more within 12 weeks of treatment. Recently the trials investigating the safety and efficacy of concizumab in hemophilia patients have been paused. The decision was based on the occurrence of thrombotic events, all not fatal, in three patients. One phase 2 trial (BAY1093884) has been also terminated because of thrombotic events.

\section{Gene Therapy}

$\mathrm{HA}$ and $\mathrm{HB}$ are monogenic, X-linked, coagulation disorders. Gene therapy may provide a long-term correction of the bleeding tendency transferring a functional copy of the gene that is required to express the missing/dysfunctional clotting factor. Several characteristics of hemophilia make the disease a good target for gene therapy: a wide range of factor level is acceptable; relatively low amount of functional protein can provide protection from spontaneous bleeding and avoid the prophylactic administration of factor concentrates; a tight control of factor level is not necessary.

Over the years the efficiency of delivery has markedly improved. The use of adeno-associated viral (AAV) vectors has so far achieved the best results in both preclinical and clinical studies. AAV vectors have been derived from wildtype AAV, a member of the parvovirus family. The specific 
characteristics that make AAV vectors a potential first choice for gene therapy is the nonpathogenic nature of wild-type $A A V$, their weak immunogenicity, and the inability to replicate autonomously. Also, they do not integrate into the host genome. The engineered vectors contain only a residual of the wild-type genetic material and most of it has been replaced with the therapeutic gene cassette. The ClinicalTrials.gov database currently lists a total of 24 active clinical trials evaluating different AVV vectors and lentivirus in HA and $\mathrm{HB}$.

BioMarin funded the first clinical trial for HA, where nine participants with severe hemophilia were enrolled into three dose cohorts using a codon-optimized AAV5 vector containing a B-domain-deleted FVIII gene. ${ }^{73}$ BioMarin has also recently submitted a Biologics License Application (BLA) to the U.S. Food and Drug Administration (FDA) for valoctoco gene roxaparvovec, its investigational AAV gene therapy, that aims at treating adult patients with HA. AAV vectors have been used in clinical trials for both HA and HB. In HB the infusion of a single dose of AAV8 vector provided long-term therapeutic FIX expression and clinical improvement. This approach, targeting hepatocytes in adult patients with $\mathrm{HB}$, has provided stable expression of FIX protein for more than 6 years. $^{74}$ The efficacy of AAV gene transfer has been enhanced through several mechanisms: better design of liverspecific promoters, codon optimization of both F8 and F9 cDNAs, and the use of engineered F8 (B-domain deleted) and F9 (gain-of-function FIX variant R338L) genes. ${ }^{75,76}$

More recently, UniQure has published data on their interim analysis of a 5-year phase $1 / 2$ clinical trial enrolling adults with $\mathrm{HB}$, showing that a single infusion of an AAV5 vector incorporating a gene cassette containing codon-optimized wild-type hFIX resulted in a stable expression of endogenous FIX for up to 1 year of follow-up. Improvement of disease severity could be observed in all participants and allowed eightof nineparticipants to discontinue FIX prophylaxis. ${ }^{77}$ of note, in contrast to data reported by other trials, no patients presented any immune response to the capsid.

\section{Challenges to Be Addressed with Novel Therapeutic Approaches to Hemophilia Treatment}

Hemophilia patients and treaters have seen the development of several exciting breakthroughs in hemophilia therapies. However, there is a significant gap of knowledge that needs to be addressed in the coming years. There is now evidence from gene therapy clinical trials to consider AAV vector gene transfer an effective option, at least in the short-to-medium term. Long-term efficacy and safety have not yet been assessed and will need to be thoroughly evaluated. The potential loss of factor expression is an important consideration. The impact of liver growth and dilution of transduced hepatocytes in younger patients is not fully understood yet. Possibly, integrating lentivirus-based strategies could overcome these obstacles. Also, it is still unclear whether the immune tolerance induction that has been achieved via AAV transfection in animal models will also translate in humans. This might be important when considering the treatment in patients with high-risk mutations for inhibitors, personal history of inhibitor, or when patients with active inhibitors are considered for gene therapy. Another restriction could be the presence of already established immunity to the viral capsid, limiting the access to this treatment or a re-dosing. However, at least for some of the gene therapy treatment options (AMT-060, AMT-061; UniQure) it seems that preexisting neutralizing antibodies to AAV5 did not compromise the sustained production of FIX in three participants.

Novel nonfactor replacement therapies, namely bispecific antibodies with FVIII mimetic properties, and drugs which affect endogenous anticoagulants such as AT and TFPI are attractive alternative approaches to the achievement of hemostasis amongst PwH. In some circumstances though, its use has been associated with thrombosis. Three thrombotic microangiopathy and twovenous thromboembolism cases have been reported under prophylaxis with emicizumab. All the thromboembolic events occurred during adjunctive treatment with aPCC for breakthrough bleeds, and specifically the thrombotic risk seems to be related to the high doses used in those cases. One fatal thrombotic event was also reported in a HA patient on a clinical trial with fitusiran, as mentioned above. The management of breakthrough bleedings at the time of concomitant nonfactor replacement therapy will become a more frequent aspect of the activities of hemophilia treaters. Also, there is very little experience with the use of nonfactor product prophylaxis and the perioperative hemostatic management. In these settings, the presence of antidotes could be helpful to effectively and safely restore the blood coagulation. At this time, no antidotes are available, except for recombinant AT, which should be able to reverse fitusiran.

Moreover, data are lacking concerning the effect of new prophylaxis regimens with nonfactor agents on the occurrence of inhibitors, considering variations in terms of timing and intensity of FVIII exposure. The achievement of immunotolerance will remain a goal of treatment, and currently there are ongoing studies aimed at exploring the possibility to achieve FVIII tolerance while obtaining protection from bleeding with emicizumab. If this approach will be proven successful, the patients that have obtained FVIII-specific tolerance could potentially switch back to factor prophylaxis. On the other hand, if the patient is to be maintained on emicizumab alone after the ITI, the duration of the immune tolerance in the absence of regular FVIII infusion is not known and potentially a break in tolerance might occur.

\section{The Evolution of Laboratory Diagnostics in Hemophilia} With the changing landscape of hemophilia care, the requirements from a laboratory diagnostic perspective have increased substantially. Here, we review the need for specialized coagulation assays in a time whenmany new hemostatic therapeutics are currently available and will become increasingly available. Of paramount importance is for laboratories to have a communication mechanism in place with clinicians to identify which hemostatic product is being used to facilitate an appropriate testing strategy to best inform clinical decision making. 


\section{Factor Assays and Extended Half-Life Products}

Critical and implicit to the proper management of patients with HA and HB is the ability to monitor FVIII and FIX levels, respectively, ${ }^{78}$ when patients receive plasma-derived or recombinant factor concentrate. There are two different functional assays to measure factor activity, the one-stage clot-based assay and the chromogenic assay, ${ }^{79}$ with the former historically being more widely used.

The clot-based assay measures how patient plasma shortens the activated partial thromboplastin time (aPTT) of FVIII or FIX-deficient plasma. The factor-deficient plasma and the patient sample are preincubated with a contact activator and phospholipids while calcium chloride is later added to initiate fibrin clot formation. ${ }^{80}$ The FVIII or FIX concentration in the patient plasma is thus presumably the rate-limiting determining factor of the aPTT.

The chromogenic assay consists of two time-dependent tests that ultimately measure FXa production, which is assumed to be proportional to the amount of FVIII or FIX present in the patient sample that is derived from a standard curve. ${ }^{79,81,82}$ Bethesda inhibitor assays, Nijmegen modified or not, are then based on the type of factor assay used in a given laboratory.

With the advent of EHL products the requirements from a diagnostic perspective have changed. Proper monitoring of FVIII and FIX with the EHL products requires chromogenic testing capacity since molecular modification and fusion with the Fc region of immunoglobulin $\mathrm{G}$ or with albumin or linkage to polyethylene glycol can lead to either over- or underestimation by approximately 20 to $50 \%$ of clotting factor activity level with many one-stage clotting assays due to interaction with the contact activator that varies from laboratory to laboratory. ${ }^{83,84}$ However,the chromogenic assays appear to consistently correlate well on external quality assessment of spiked samples with a variety of EHLs. ${ }^{80}$ The discrepancy that occurs with aPTT-based assays can be of clinical importance in patients being treated for a major bleed, trauma, or surgery, and thus it is now important to have chromogenic testing capacity to best guide clinical care. ${ }^{80}$

\section{Laboratory Monitoring of Emicizumab}

Dosing of emicizumab is based on weight and can occur at frequency ranging from weekly to monthly. Emicizumab drug concentration assays may prove useful in defining which patients can be converted to less frequent dosing strategies particularly since pharmacokinetic data demonstrate a linear relationship between steady state trough plasma emicizumab concentrations and bleeding rates. ${ }^{85}$

Emicizumab interacts with all intrinsic pathway clottingbased laboratory assays, including all aPTT-based assays, rendering them unreliable and potentially falsely reassuring to the unaware provider. The binding characteristics of emicizumab are such that even low plasma concentrations normalize the aPTT. Under normal circumstances, aPTTbased clotting assays measure the total clotting time of the intrinsic pathway of coagulation, including activation of FVIII to FVIIIa by thrombin; emicizumab does not require activation by thrombin and will therefore result in an exceedingly short clotting time. Therefore, a one-stage assay performed in the presence of emicizumab would grossly overestimate FVIII:C. ${ }^{86}$

On the other hand, the FVIII chromogenic assay will provide an accurate measure of FVIII activity in a sample containing emicizumab. FVIII chromogenic assay measures the FVIII-dependent activation of FX using purified bovine or human coagulation factor. In the first stage of this assay, patient plasma is added to a reaction mixture that usually contains FIXa, FX, calcium ions, phospholipids, and trace amounts of thrombin. Thrombin triggers the activation of FVIII and the subsequent FIXa-mediated activation of FX. In contrast to the FVIII aPTT-based clotting assay, the addition of thrombin in the first step means that activation of FVIII to FVIIla is not a major influencing determinant of the assay. FXa production is presumed to be proportional to the FVIII concentration in the plasma sample. Since emicizumab selectively binds to human FIXa and FX, a FVIII chromogenic assay based on bovine factors is recommended. ${ }^{87}$

Neutralizing anti-emicizumab antibody interfering with the drug's hemostatic efficacy has been rarely described, ${ }^{88}$ thus the ability to measure emicizumab antibodies using enzyme-linked-immunosorbent serologic assay (ELISA)based methodology is important in cases where clinical hemostasis is called to question. ${ }^{87}$

Important consideration must be given to the FVIII assay and Bethesda assay based on it in patients receiving emicizumab, as one-stage aPTT assays will substantially overestimate the FVIII due to the effect of emicizumab on clotbased assays. ${ }^{85,89}$ This is an important consideration as patients on emicizumab may occasionally require FVIII replacement for major bleeds or surgeries. Also, others with allo-inhibitors may concomitantly be treated with immune tolerance induction therapy where accurate monitoring of FVIII and FVIII inhibitor levels will be necessary. In these cases, a chromogenic FVIII assay using bovine components must be used as the bovine proteins (FIX and FX) are insensitive to emicizumab while still sensitive to human FVIII rendering ability to measure endogenous or infused FVIII. $^{90}$

\section{Laboratory Monitoring of Fitusiran and Concizumab}

Plasma AT measurements in ongoing clinical trials with fitusiran are measured by a chromogenic AT assay while global hemostasis is being evaluated with an automated thrombogram. ${ }^{68,69}$ Concizumab appears procoagulant in vitro as measured by D-dimer and prothrombin fragments as well as global hemostatic assays. ${ }^{91}$ The optimal method for monitoring these classes of hemostatic drugs however remains to be determined and should become clearer with ongoing clinical trials. ${ }^{80}$

\section{Dynamic Assays of Coagulation}

Dynamic assays such as thrombin generation and viscoelastic (thromboelastography and rotational thromboelastrometry) assays will likely play an increasing role in the care of $\mathrm{PwH}$ given the increasing array of products available with hemostatic effect ( - Fig. 1). In fact, the International Society 


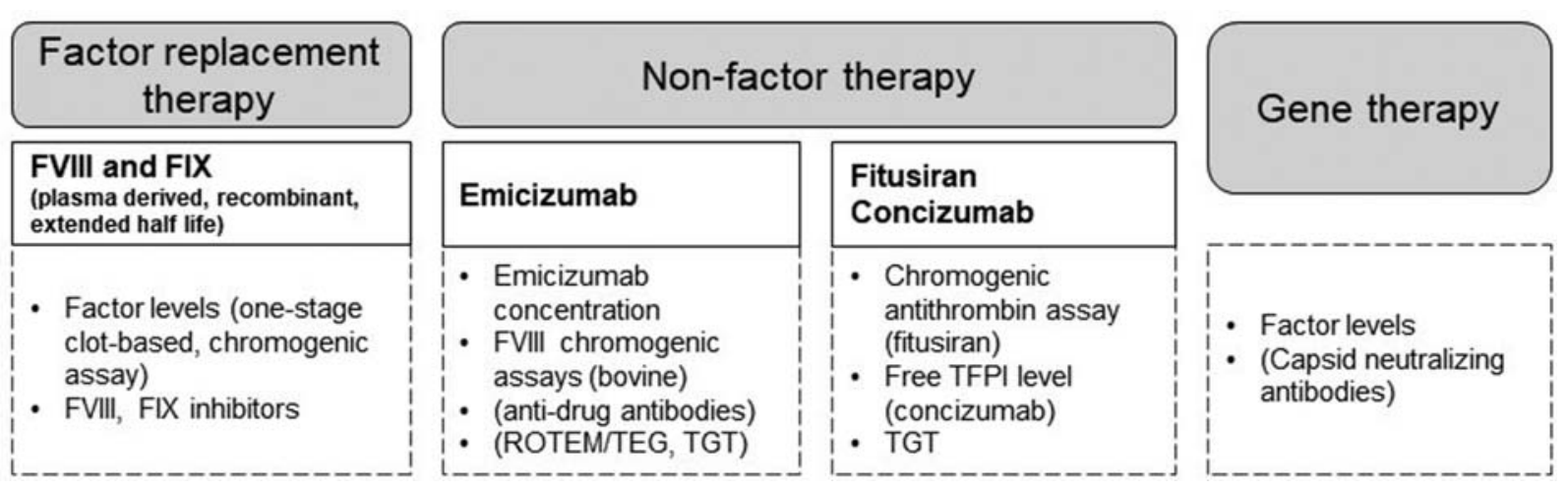

Fig. 1 Monitoring of novel hemophilia therapies. FVIII, factor VIII; FIX, factor IX; ROTEM, rotational thromboelastometry; TGT, thrombin generation tests; TEG, thromboelastography; TFPI, tissue factor pathway inhibitor.

on Thrombosis and Haemostasis released a statement supporting the use of viscoelastic assays in clinical management and clinical trials of $\mathrm{PwH}^{92}$

\section{Gaps of Knowledge}

In the era of rapidly evolving therapies for hemophilia, there are new challenges and opportunities for the special coagulation laboratory and clinician. Specialized testing capacity (whether local or remote via reference) is important but only if the test is properly matched to the hemostatic therapeutic and clinical scenario. Thus, effective communication with the laboratory is important now more than ever. The current unknowns or unmet needs from a laboratory perspective include developing a firm understanding of the clinical significance of emicizumab drug levels in the real world, expanding and understanding the utility of dynamic assays of coagulation with novel hemostatic agents as well as solidifying knowledge-translation and exchange efforts to facilitate proper appropriation of laboratory tests for given hemostatic agents.

\section{Time Capsule}

- FVIII/FIX concentrates will remain the standard of care in most patients worldwide and achievement of immunotolerance will still be a goal of treatment.

- Hemophilia-related fatality will decrease in the first years of life thanks to early primary prophylaxis with nonintravenous products.

- Effective communication with the laboratory to ensure proper test appropriation will be critical given the availability of extended half-life factor and nonfactor products.

- Gene therapy will be optimized to achieve long-term factor production and strategies to allow for re-dosing will be devised.

\section{Conflict of Interest}

Dr. Matino reports personal fees from Sanofi, grants and personal fees from Sobi, personal fees from UBS, grants and personal fees from Pfizer, personal fees from NovoNordisk, personal fees from BIOVIIIX, outside the submitted work.

\section{Authors}

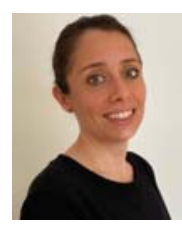

\section{Alice Trinchero}

Alice Trinchero, MD, completed her residency in Internal Medicine at the University of Pavia, Italy. She obtained the German subspecialization in Hämostaseologie at the Johannes Gutenberg University Mainz, Germany, where she worked at the outpatient clinic of the Center for Thrombosis and Hemostasis between 2015 and 2020. She is involved in clinical research projects on venous thromboembolism and rare hemostasis disorders. She currently serves as Staff Physician at the Department of Hematology-Oncology of the University Hospital Zurich, Switzerland, where she is involved in the care of patients with coagulation disorders.

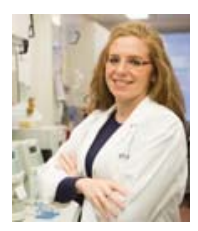

\section{Michelle Sholzberg}

Dr. Michelle Sholzberg is a hemostasis-focused hematologist and clinical epidemiologist with a focus on clinical trials. She is the Medical Director of the Coagulation Laboratory at St. Michael's Hospital, the Director of the Hematology-Oncology Clinical Research Group and of the Hematology-Immunology Translational Research Theme. Her research interests include inherited and acquired disorders of hemostasis. Currently, she is involved in the study of: COVID-19 coagulopathy, prediction tools for perioperative bleeding, acquired hemophilia, the intersection of women's health and bleeding disorders, and new treatments for immune thrombocytopenia.

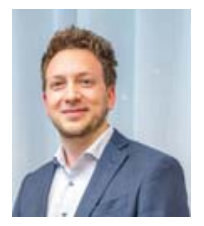

\section{Davide Matino}

Dr. Davide Matino, MD, had his residency training at the University of Rome "Tor Vergata" in 2014. He completed a Clinical Fellowship in bleeding disorders at McMaster University where he also received his MSc in Health Research bleeding Methodology, defending his thesis "Clinical application of the Web-Accessible Population Pharmacokinetic Service - Hemophilia (WAPPS-Hemo): proposal for a pilot study of a population pharmacokinetic approach to tailored prophylaxis in hemophilia". He then joined the 
University of Perugia as Assistant Professor in Pharmacology working on a research project focused on the study of the immune response to FVIII in hemophilia, funded through a Young Investigator Award from the Italian Ministry of Health. His research is focused on inhibitor development in hemophilia, personalized-prophylaxis regimens in hemophilia, pharmacokinetic of clotting factor concentrates and the treatment of hemophilia patients with inhibitors. He is actively involved in several industry-sponsored and investigator initiated clinical trials in hemophilia, including new extended half-life products, gene therapy and non-factor replacement therapy.

\section{References}

1 Mannucci PM, Tuddenham EG. The hemophilias-from royal genes to gene therapy. N Engl J Med 2001;344(23):1773-1779

2 Berntorp E, Shapiro AD. Modern haemophilia care. Lancet 2012; 379(9824):1447-1456

3 Melchiorre D, Linari S, Manetti M, et al. Clinical, instrumental, serological and histological findings suggest that hemophilia $B$ may be less severe than hemophilia A. Haematologica 2016;101 (02):219-225

4 Makris M, Oldenburg J, Mauser-Bunschoten EP, Peerlinck K, Castaman G, Fijnvandraat K; Subcommittee on Factor VIII, Factor IX and Rare Bleeding Disorders. The definition, diagnosis and management of mild hemophilia A: communication from the SSC of the ISTH. J ThrombHaemost 2018;16(12):2530-2533

5 Pool JG, Gershgold EJ, Pappenhagen AR. High-potency antihaemophilic factor concentrate prepared from cryoglobulin precipitate. Nature 1964;203:312

6 Soucie JM, Symons J IV, Evatt B, Brettler D, Huszti H, Linden J; Hemophilia Surveillance System Project Investigators. Homebased factor infusion therapy and hospitalization for bleeding complications among males with haemophilia. Haemophilia 2001;7(02):198-206

7 Larsson SA, Wiechel B. Deaths in Swedish hemophiliacs, 19571980. Acta Med Scand 1983;214(03):199-206

8 Larsson SA. Life expectancy of Swedish haemophiliacs, 18311980. Br J Haematol 1985;59(04):593-602

9 Ikkala E, Helske T, Myllylä G, Nevanlinna HR, Pitkänen P, Rasi V. Changes in the life expectancy of patients with severe haemophilia A in Finland in 1930-79. Br J Haematol 1982;52(01):7-12

10 Smit C, Rosendaal FR, Varekamp I, et al. Physical condition, longevity, and social performance of Dutch haemophiliacs, 1972-85. BMJ 1989;298(6668):235-238

11 Chorba TL, Holman RC, Strine TW, Clarke MJ, Evatt BL. Changes in longevity and causes of death among persons with hemophilia A. Am J Hematol 1994;45(02):112-121

12 Fischer K, van der Bom JG, Molho P, et al. Prophylactic versus ondemand treatment strategies for severe haemophilia: a comparison of costs and long-term outcome. Haemophilia 2002;8(06):745-752

13 Evatt BL, Gomperts ED, McDougal JS, Ramsey RB. Coincidental appearance of LAV/HTLV-III antibodies in hemophiliacs and the onset of the AIDS epidemic. N Engl J Med 1985;312(08):483-486

14 Mannucci PM. Back to the future: a recent history of haemophilia treatment. Haemophilia 2008;14(Suppl 3):10-18

15 Oldenburg J, Dolan G, Lemm G. Haemophilia care then, now and in the future. Haemophilia 2009;15(Suppl 1):2-7

16 Soucie JM, Nuss R, Evatt B, et al; The Hemophilia Surveillance System Project Investigators. Mortality among males with hemophilia: relations with source of medical care. Blood 2000;96(02): 437-442

17 Chorba TL, Holman RC, Clarke MJ, Evatt BL. Effects of HIV infection on age and cause of death for persons with hemophilia $\mathrm{A}$ in the United States. Am J Hematol 2001;66(04):229-240
18 Wood WI, Capon DJ, Simonsen CC, et al. Expression of active human factor VIII from recombinant DNA clones. Nature 1984; 312(5992):330-337

19 Franchini M, Mannucci PM. Hemophilia A in the third millennium. Blood Rev 2013;27(04):179-184

20 Grushin K, Miller J, Dalm D, et al. Lack of recombinant factor VIII Bdomain induces phospholipid vesicle aggregation: implications for the immunogenicity of factor VIII. Haemophilia 2014;20(05): 723-731

21 Wight J, Paisley S. The epidemiology of inhibitors in haemophilia A: a systematic review. Haemophilia 2003;9(04):418-435

22 Male C, Andersson NG, Rafowicz A, et al; PedNetStudy Group. Inhibitor incidence in an unselected cohort of previously untreated patients with severe haemophilia B: a PedNet study. Haematologica 2020 (e-pub ahead of print) . Doi: 10.3324/haematol.2019.239160

23 Gringeri A, Mantovani LG, Scalone L, Mannucci PM; COCIS Study Group. Cost of care and quality of life for patients with hemophilia complicated by inhibitors: the COCIS Study Group. Blood 2003; 102(07):2358-2363

24 Walsh CE, Jiménez-Yuste V, Auerswald G, Grancha S. The burden of inhibitors in haemophilia patients. ThrombHaemost 2016;116 (Suppl 1):S10-S17

25 Giles AR, Mann KG, Nesheim ME. A combination of factor Xa and phosphatidylcholine-phosphatidylserine vesicles bypasses factor VIII in vivo. Br J Haematol 1988;69(04):491-497

26 Ananyeva NM, Lee TK, Jain N, Shima M, Saenko EL. Inhibitors in hemophilia $\mathrm{A}$ : advances in elucidation of inhibitory mechanisms and in inhibitor management with bypassing agents. SeminThrombHemost 2009;35(08):735-751

27 Srivastava A, Brewer AK, Mauser-Bunschoten EP, et al; Treatment Guidelines Working Group on Behalf of The World Federation of Hemophilia. Guidelines for the management of hemophilia. Haemophilia 2013;19(01):e1-e47

28 Manco-Johnson MJ, Kempton CL, Reding MT, et al. Randomized, controlled, parallel-group trial of routine prophylaxis vs. ondemand treatment with sucrose-formulated recombinant factor VIII in adults with severe hemophilia A (SPINART). J ThrombHaemost 2013;11(06):1119-1127

29 Kavakli K, Yang R, Rusen L, Beckmann H, Tseneklidou-Stoeter D, Maas Enriquez M; LEOPOLD II Study Investigators. Prophylaxis vs. on-demand treatment with BAY 81-8973, a full-length plasma protein-free recombinant factor VIII product: results from a randomized trial (LEOPOLD II). J ThrombHaemost 2015;13(03): 360-369

30 Tagliaferri A, Feola G, Molinari AC, et al; POTTER Study Group. Benefits of prophylaxis versus on-demand treatment in adolescents and adults with severe haemophilia A: the POTTER study. ThrombHaemost 2015;114(01):35-45

31 Valentino LA, Mamonov V, Hellmann A, et al; Prophylaxis Study Group. A randomized comparison of two prophylaxis regimens and a paired comparison of on-demand and prophylaxis treatments in hemophilia A management. J ThrombHaemost 2012;10 (03):359-367

32 Gringeri A, Lundin B, von Mackensen S, Mantovani L, Mannucci PM; ESPRIT Study Group. A randomized clinical trial of prophylaxis in children with hemophilia A (the ESPRIT Study). J ThrombHaemost 2011;9(04):700-710

33 O'Hara J, Sima CS, Frimpter J, Paliargues F, Chu P, Presch I. Long-term outcomes from prophylactic or episodic treatment of haemophilia A: a systematic review. Haemophilia 2018;24(05):e301-e311

34 Hilliard P, Zourikian N, Blanchette V, et al. Musculoskeletal health of subjects with hemophilia A treated with tailored prophylaxis: Canadian Hemophilia Primary Prophylaxis (CHPS) Study. J ThrombHaemost 2013;11(03):460-466

35 Witmer C, Presley R, Kulkarni R, Soucie JM, Manno CS, Raffini L. Associations between intracranial haemorrhage and prescribed prophylaxis in a large cohort of haemophilia patients in the United States. Br J Haematol 2011;152(02):211-216 
36 Tagliaferri A, Rivolta GF, Iorio A, et al; Italian Association of Hemophilia Centers. Mortality and causes of death in Italian persons with haemophilia, 1990-2007. Haemophilia 2010;16 (03):437-446

37 Triemstra M, Rosendaal FR, Smit C, Van der Ploeg HM, Briët E. Mortality in patients with hemophilia. Changes in a Dutch population from 1986 to 1992 and 1973 to 1986 . Ann Intern Med 1995;123(11):823-827

38 Plug I, Van Der Bom JG, Peters M, et al. Mortality and causes of death in patients with hemophilia, 1992-2001: a prospective cohort study. J ThrombHaemost 2006;4(03):510-516

39 Darby SC, Kan SW, Spooner RJ, et al. Mortality rates, life expectancy, and causes of death in people with hemophilia A or B in the United Kingdom who were not infected with HIV. Blood 2007;110 (03):815-825

40 Chang CY, Yeh GC, Lin SY, Tseng IJ, Tsai CH, Lee YW. Trends in the epidemiology, diagnosed age and mortality rate of haemophiliacs in Taiwan: a population-based study, 1997-2009. Haemophilia 2014;20(04):535-540

41 Eckhardt CL, Loomans JI, van Velzen AS, et al; INSIGHT Study Group. Inhibitor development and mortality in non-severe hemophilia A. J ThrombHaemost 2015;13(07):1217-1225

42 Goel R, Krishnamurti L. Mortality, health care utilization and associated diagnoses in hospitalized patients with haemophilia in the United States: first reported nationwide estimates. Haemophilia 2012;18(05):688-692

43 Lövdahl S, Henriksson KM, Baghaei F, et al. Incidence, mortality rates and causes of deaths in haemophilia patients in Sweden. Haemophilia 2013;19(03):362-369

44 Walsh CE, Soucie JM, Miller CH; United States Hemophilia Treatment Center Network. Impact of inhibitors on hemophilia A mortality in the United States. Am J Hematol 2015;90(05):400-405

45 Yoo KY, Kim SK, Kwon SS, et al. Life expectancy of Korean haemophiliacs, 1991-2012. Haemophilia 2014;20(04):e356-e358

46 Mansouritorghabeh H, Rahimi H, Mohades ST, Behboudi M. Causes of death among 379 patients with hemophilia: a developing country's report. Clin Appl ThrombHemost 2018;24(04): 612-617

47 Loomans JI, Eckhardt CL, Reitter-Pfoertner SE, et al. Mortality caused by intracranial bleeding in non-severe hemophilia A patients. J ThrombHaemost 2017;15(06):1115-1122

48 Jardim LL, van der Bom JG, Caram-Deelder C, Gouw SC, Leal Cherchiglia M, Meireles Rezende S. Mortality of patients with haemophilia in Brazil: first report. Haemophilia 2019;25(03): e146-e152

49 Callaghan MU, Sidonio R, Pipe SW. Novel therapeutics for hemophilia and other bleeding disorders. Blood 2018;132(01):23-30

50 Hart DP, Kessler CM, Aledort L. Re-personalization and stratification of hemophilia care in an evolving treatment landscape. Hematology 2019;24(01):737-741

51 Oldenburg J. Optimal treatment strategies for hemophilia: achievements and limitations of current prophylactic regimens. Blood 2015;125(13):2038-2044

52 Ling M, Heysen JP, Duncan EM, Rodgers SE, Lloyd JV. High incidence of ankle arthropathy in mild and moderate haemophilia A. ThrombHaemost 2011;105(02):261-268

53 Soucie JM, Monahan PE, Kulkarni R, Konkle BA, Mazepa MA; US Hemophilia Treatment Center Network. The frequency of joint hemorrhages and procedures in nonsevere hemophilia A vs B. Blood Adv 2018;2(16):2136-2144

54 Aronstam A, Arblaster PG, Rainsford SG, et al. Prophylaxis in haemophilia: a double-blind controlled trial. Br J Haematol 1976; 33(01):81-90

55 Nilsson IM, Hedner U, Ahlberg A. Haemophilia prophylaxis in Sweden. Acta PaediatrScand 1976;65(02):129-135

56 Iorio A, Iserman $\mathrm{E}$, Blanchette $\mathrm{V}$, et al. Target plasma factor levels for personalized treatment in haemophilia: a Delphi consensus statement. Haemophilia 2017;23(03):e170-e179
57 Gouw SC, van der Bom JG, Ljung R, et al; PedNet and RODIN Study Group. Factor VIII products and inhibitor development in severe hemophilia A. N Engl J Med 2013;368(03):231-239

58 Peyvandi F, Mannucci PM, Garagiola I, et al. A randomized trial of factor VIII and neutralizing antibodies in hemophilia A. N Engl J Med 2016;374(21):2054-2064

59 Oldenburg J, Austin SK, Kessler CM. ITI choice for the optimal management of inhibitor patients - from a clinical and pharmacoeconomic perspective. Haemophilia 2014;20(Suppl 6):17-26

60 Gringeri A. VWF/FVIII concentrates in high-risk immunotolerance: the RESIST study. Haemophilia 2007;13(Suppl 5):73-77

61 Kitazawa T, Esaki K, Tachibana T, et al. Factor VIIIa-mimetic cofactor activity of a bispecific antibody to factors IX/IXa and $\mathrm{X} / \mathrm{Xa}$, emicizumab, depends on its ability to bridge the antigens. ThrombHaemost 2017;117(07):1348-1357

62 Oldenburg J, Mahlangu JN, Kim B, et al. Emicizumab prophylaxis in hemophilia A with inhibitors. N Engl J Med 2017;377(09): 809-818

63 Young G, Liesner R, Chang T, et al. A multicenter, open-label phase 3 study of emicizumab prophylaxis in children with hemophilia $A$ with inhibitors. Blood 2019;134(24):2127-2138

64 Mahlangu J, Oldenburg J, Paz-Priel I, et al. Emicizumab prophylaxis in patients who have hemophilia A without inhibitors. $\mathrm{N}$ Engl J Med 2018;379(09):811-822

65 Sehgal A, Barros S, Ivanciu L, et al. An RNAi therapeutic targeting antithrombin to rebalance the coagulation system and promote hemostasis in hemophilia. Nat Med 2015;21(05):492-497

66 Bolliger D, Szlam F, Suzuki N, Matsushita T, Tanaka KA. Heterozygous antithrombin deficiency improves in vivo haemostasis in factor VIIIdeficient mice. ThrombHaemost 2010;103(06):1233-1238

67 Safdar H, Cheung KL, Salvatori D, et al. Acute and severe coagulopathy in adult mice following silencing of hepatic antithrombin and protein C production. Blood 2013;121(21):4413-4416

68 Pasi KJ, Rangarajan S, Georgiev P, et al. Targeting of antithrombin in hemophilia A or B with RNAi therapy. N Engl J Med 2017;377 (09):819-828

69 Pasi KJ, Georgiev P, Mant T, et al. Fitusiran, an investigational RNAi therapeutic targeting antithrombin for the treatment of hemophilia: updated results from a phase 1 and phase $1 / 2$ extension study in patients with inhibitors. Blood 2016;128(22):1397

70 Hemophilia WFo. Update: FDA lifts suspension of fitusiran trial [cited 20186 March]. Available at: https://news.wfh.org/updatefda-lifts-suspension-fitusiran-trial/

71 Hilden I, Lauritzen B, Sørensen BB, et al. Hemostatic effect of a monoclonal antibody mAb 2021 blocking the interaction between FXa and TFPI in a rabbit hemophilia model. Blood 2012; 119(24):5871-5878

72 Shapiro AD, Angchaisuksiri P, Astermark J, et al. Subcutaneous concizumab prophylaxis in hemophilia $A$ and hemophilia $A / B$ with inhibitors: phase 2 trial results. Blood 2019;134(22):1973-1982

73 Rangarajan S, Walsh L, Lester W, et al. AAV5-factor VIII gene transfer in severe hemophilia A. N Engl J Med 2017;377(26):2519-2530

74 Nathwani AC, Reiss UM, Tuddenham EG, et al. Long-term safety and efficacy of factor IX gene therapy in hemophilia B. N Engl J Med 2014;371(21):1994-2004

75 Nathwani AC, Tuddenham EG, Rangarajan S, et al. Adenovirusassociated virus vector-mediated gene transfer in hemophilia B. N Engl J Med 2011;365(25):2357-2365

76 McIntosh J, Lenting PJ, Rosales C, et al. Therapeutic levels of FVIII following a single peripheral vein administration of rAAV vector encoding a novel human factor VIII variant. Blood 2013;121(17): 3335-3344

77 Miesbach W, Meijer K, Coppens M, et al. Gene therapy with adeno-associated virus vector 5-human factor IX in adults with hemophilia B. Blood 2018;131(09):1022-1031

78 Jennings I, Kitchen DP, Woods TAL, Kitchen S, Walker ID, Preston FE. Laboratory performance in the World Federation of Hemophilia EQA programme, 2003-2008. Haemophilia 2009;15(02):571-577 
79 Potgieter JJ, Damgaard M, Hillarp A. One-stage vs. chromogenic assays in haemophilia A. Eur J Haematol 2015;94(Suppl 77): 38-44

80 Teichman J, Chaudhry HR, Sholzberg M. Novel assays in the coagulation laboratory: a clinical and laboratory perspective. Transfus Apheresis Sci 2018;57(04):480-484

81 Moser KA, Adcock Funk DM. Chromogenic factor VIII activity assay. Am J Hematol 2014;89(07):781-784

82 Peyvandi F, Oldenburg J, Friedman KD. A critical appraisal of onestage and chromogenic assays of factor VIII activity. J ThrombHaemost 2016;14(02):248-261

83 Kitchen S, Kershaw G, Tiefenbacher S. Recombinant to modified factor VIII and factor IX - chromogenic and one-stage assays issues. Haemophilia 2016;22(Suppl 5):72-77

84 Bowyer AE, Hillarp A, Ezban M, Persson P, Kitchen S. Measuring factor IX activity of nonacog beta pegol with commercially available one-stage clotting and chromogenic assay kits: a twocenter study. J ThrombHaemost 2016;14(07):1428-1435

85 Shima $M$, Hanabusa $H$, Taki $M$, et al. Long-term safety and efficacy of emicizumab in a phase $1 / 2$ study in patients with hemophilia A with or without inhibitors. Blood Adv 2017;1(22): 1891-1899

86 Al-Samkari H, Croteau SE. Shifting landscape of hemophilia therapy: implications for current clinical laboratory coagulation assays. Am J Hematol 2018;93(08):1082-1090
87 Müller J, Pekrul I, Pötzsch B, Berning B, Oldenburg J, Spannagl M. Laboratory monitoring in emicizumab-treated persons with hemophilia A. ThrombHaemost 2019;119(09):1384-1393

88 National Hemophilia Foundation. MASAC Safety Information Update on Emicizumab (HEMLIBRA) 2018. Available at: https://www. hemophilia.org/Newsroom/Medical-News/MASAC-Safety-Information-Update-on-Emicizumab-HEMLIBRA. Accessed May 27, 2020

89 Dargaud Y, Lienhart A, Janbain M, Le Quellec S, Enjolras N, Negrier $C$. Use of thrombin generation assay to personalize treatment of breakthrough bleeds in a patient with hemophilia and inhibitors receiving prophylaxis with emicizumab. Haematologica 2018; 103(04):e181-e183

90 Calatzis AKN, Levy G, Adamkewitcz J, Eds. Effect of emicizumab a humanized bispecific antibody mimicking FVIII cofactor function - on a variety of assay systems [Abstract]. Paper presented at: European Congress on Thrombosis and Haemostasis Abstract; 2016; The Hague

91 Chowdary P, Lethagen S, Friedrich U, et al. Safety and pharmacokinetics of anti-TFPI antibody (concizumab) in healthy volunteers and patients with hemophilia: a randomized first human dose trial. J Thromb Haemost 2015;13(05):743-754

92 Chitlur M, Rivard GE, Lillicrap D, et al. Recommendations for Performing Thromboelastography/Thromboelastometry in Hemophilia: Communication From the SSC of the ISTH. J Thromb Haemost 2014;12(01):103-106 